\title{
New Undergraduate Courses in Control
}

\author{
Jan Tommy Gravdahl and Olav Egeland
}

Department of Engineering Cybernetics

Norwegian University of Science and Technology

N-7491 Trondheim, Norway

email: \{Tommy.Gravdahl, Olav.Egeland\}@itk.ntnu.no

fax: +47 73594399

June 17, 2004 


\section{Introduction}

Control education at the Norwegian University of Science and Technology (NTNU) is provided by the Department of Engineering Cybernetics in the Faculty of Information Technology, Mathematics, and Electrical Engineering. Each year the department graduates about 100 students with Master's degrees. The department offers an integrated 5-year degree where the students are recruited directly to control studies in their first year. Our success in recruitment is partly due, we believe, to our use of the word cybernetics in the name of the department. Although this term is not widely used in the international control community, potential students apparently find it exciting.

At the undergraduate level, control education has been heavily influenced by the traditional electrical engineering curriculum. Consequently, many of our undergraduate students have had limited training in mechanical and chemical engineering. In addition, students who specialize in motion control or chemical process control are expected to master advanced mathematical modeling to meet the expectations of industry. In response to these needs, a new course in modeling and simulation is now taught in the third year to provide students with the required background. 
The department has also responded to a recent trend in which students generally require motivation in the form of problem-driven education. For the last two years of their studies, we have therefore introduced more project work in the courses, and extended the duration of the student project to two months and the thesis work to five months. However, our main innovation is the new introductory course Introduction to Computerized Control, developed for the first year of study. Although it is a challenge to introduce the basic concepts of control engineering at this level, our experience has been positive, and we believe that this course is necessary for motivating the students to complete the many theoretical courses at the undergraduate level.

The control-related courses in the master's program are listed in Table 1. In addition to these topics, there are compulsory courses such as mathematics, statistics, and circuit analysis. Each course comprises 7.5 credits, and students are required to take 60 credits per year. In the Norwegian system there are two semesters per year, and each semester comprises four courses.

The modeling and simulation course is taught in the third year of undergraduate study. The students have taken a basic course in automatic control theory in their fourth semester, and they have had standard undergradu- 


\begin{tabular}{|l||l|}
\hline Year & Course \\
\hline \hline 1 st & Introduction to Computerized Control \\
\hline 2nd & Control Engineering, Computerized Control in Industrial Systems \\
\hline 3rd & Linear System Theory, Modeling and Simulation \\
& Optimization and Control, Real-Time Systems \\
\hline 4 th & The students specialize in either motion control, \\
& process control, or instrumentation and computer systems \\
\hline 5 th & Project work and MSc Thesis \\
\hline
\end{tabular}

Table 1: Control-related courses in the Department of Engineering Cybernetics, Norwegian University of Science and Technology. The courses Introduction to Computerized Control and Modeling and Simulation were recently developed to meet the special needs of the curriculum. 
ate courses in engineering mathematics. The curriculum in the fourth and fifth years was developed to achieve specialization in either motion control, process control, or instrumentation and computer systems. According to the area of specialization, the students can choose courses such as nonlinear systems, guidance and control, embedded computer systems, modeling and identification of biological systems, instrumentation systems, stochastic and adaptive systems, and robust control.

In their final year, the students do their project work and five months of thesis work. In parallel with the project work, the students must take two subjects as guided self study. These topics include robotics, nonlinear motion control, servomechanisms, model predictive control and optimization, real time theory, system identification and adaptive control, and Kalman filtering.

The focus of this article is on our two new undergraduate courses Introduction to Computerized Control and Modeling and Simulation. 


\section{Introduction to Computerized Control}

\section{Background}

The basic control engineering course is given at the end of the second year, and this class is the first experience the students have traditionally had with the concept of automatic control. Since some students drop out before they reach this level, we have introduced an introductory control course in the first year. The idea is to introduce some of the topics that will appear later in other courses. It is also our hope that this approach will motivate students to complete the theoretical courses during their undergraduate studies.

Introduction to Computerized Control presents dynamics, time-domain control design, instrumentation, and real-time computing in the format of four hours of lectures per week, two compulsory laboratory exercises, and seven web-based sets of problems. The course gives insight into key problems in control theory, instrumentation, and other topics that form the basis for implementing automatic control systems. While it is too early to evaluate the effect of this course, the response from the students has been positive, and comments such as "This is the most interesting subject we have had so far" are typical. The course is taught during fourteen weeks of lectures, and is divided into two distinct parts, described below. 


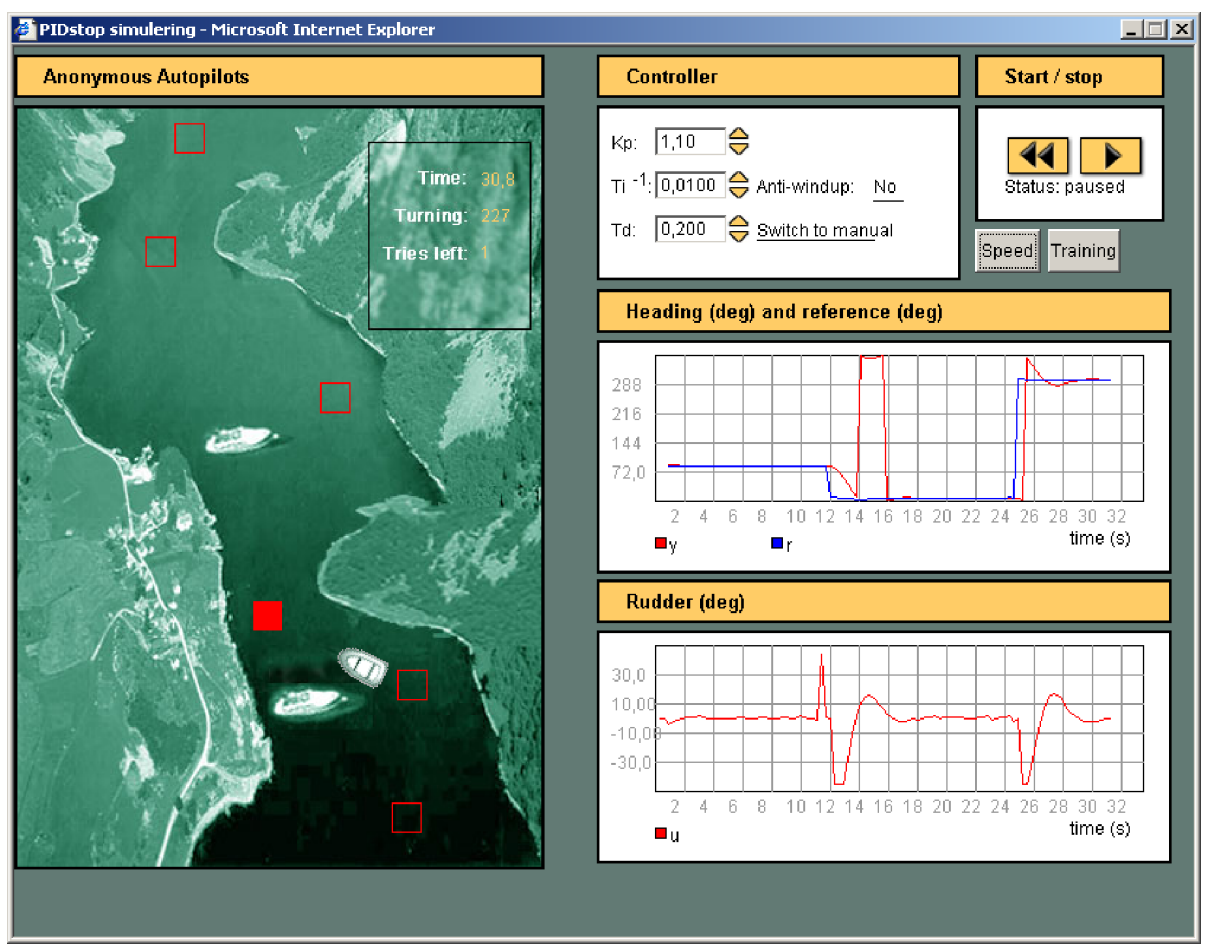

Figure 1: Tuning of a PID autopilot. In this exercise, the students tune a PID control law for a ship autopilot. The goal of the exercise is to ensure that the ship navigates a fjord by means of a set of way-points in the shortest possible time without hitting the shore or other vessels. The simulator requires only Java applets, and runs on standard computers. If the autopilot is well tuned, the speed of the ship can be increased. The students compete against each other in navigating the fjord in the shortest possible time. A high score list is kept to stimulate competition. The simulator is part of PIDstop, a system developed by Tore Bjølseth, a research assistent at NTNU. 


\section{Control fundamentals}

The first half of Introduction to Computerized Control covers the terminology of control systems, and includes an introduction to differential equations and dynamic systems. Balance laws are introduced, and mathematical models of various physical processes are derived. First and second-order models of electrical, thermal, mechanical, and fluid systems are covered. Control and simulation of these models is then demonstrated. All control design is done in the time domain, and PID control is applied to temperature control, fluid-level control, cruise control, and spacecraft attitude control. We present simulation by Euler's method, with the understanding that many systems require more advanced simulation methods that will be taught during the Modeling and Simulation course. The students are introduced to block diagrams, and are shown how to implement block diagrams in Simulink.

The first part of the course ends with a midterm exam and a group project. In the project, the students work for two weeks in groups of four to find a solution to a rather loosely stated control problem. An example of one such problem statement is "Develop an adaptive cruise control for cars." The results from the project are presented in front of the class. Topics discussed in the presentation include modeling, control design and tuning, simulation, and analysis 


\section{Implementation aspects of control systems}

The second half of the course covers implementation aspects of control systems. The students are introduced to sensor principles based on resistance, capacitance, light, and magnetism. These principles are applied to the measurement of position, velocity, force, and temperature. Actuators such as control valves and electrical motors are described. Laboratory assignments on flow measurement and angle measurement are used to support this part of the curriculum. For flow measurement the students perform experiments on a laboratory test rig, and compare measurements based on principles such as orifice flow, Coriolis effect, turbine meters, vortex meters, and electromagnetic flowmeters. Angles are measured on a dc motor using different angle transducers.

Finally, real-time systems and real-time programming are briefly presented, and there is a two-hour lecture on schedulers, synchronization, and state machines. Further topics in this part of the course are logic control, logic circuits, Boolean algebra, PLC, A/D and D/A converters, sampling, scaling, calibration, and filters. 


\section{Textbook}

In the process of developing this course we were unable to find a textbook covering all of the topics at an introductory level. As a result, we are currently developing our own material on dynamics, control systems, real-time computing, and logic control. The instrumentation and electronics topics rely on textbooks and older lecture notes written at the department. We are currently compiling this material into a new textbook for the course. The language of the book will be Norwegian. More information on the course can be found in [1]. 


\section{Modeling and Simulation}

Modeling and simulation of dynamic processes has developed from a tool for specialists in universities and industry to a routine activity in many sectors of industry and in entertainment. Teenagers are familiar with animation in computer games, and it is no longer difficult to explain the concept and need for real-time simulation. Industrial customers of control systems increasingly require detailed simulation studies to document system performance, and elaborate simulators with associated real-time animation are used in sales and training. It is interesting to consider the implications of these developments for the teaching of modeling and simulation to students specializing in control.

Control students have traditionally been among the few students who receive education in mathematical modeling and simulation. Modeling and simulation courses have focused on generic model representations and inputoutput models. The physical plants and simulation techniques presented have, to a large extent, been trivial. This level of teaching was sufficient five to ten years ago in relation to the needs of industry and research labs at that time. In contrast, there are many students outside of the control area who now master modeling and simulation, and control-oriented simulation mod- 
els used in industry are becoming highly specialized and detailed. Although advanced mathematical models for use in simulators are usually available in different branches of the engineering literature, the relevant books and university courses are oriented toward static models or the use of the Fourier transform, rather than state-space models and the Laplace transform, which are the main tools of the control engineer. As a result, several courses or books must be consulted to obtain relevant modeling information for a particular process. To meet this challenge it is important to improve the training in modeling and simulation so that students who specialize in control can provide the expertise required by industrial companies and research institutions.

In response to this situation, the modeling and simulation course offered to the control students at NTNU has been updated, and a new textbook has been developed [2]. The style of modeling in the course is largely adopted from robotics courses [3], [4], [5], where modeling is presented in detail and in a precise style based on equations. Generic modeling tools such as network descriptions, energy-based methods, and passivity are briefly introduced, while it is assumed that the students have a background in state space models and the Laplace transform from a basic course in control theory. The main part of the course focuses on the detailed modeling of electromechanical systems, hydraulic systems, friction, rigid body kinematics, equations of 
motion, balance equations for mass, momentum and energy, and isentropic gas dynamics.

The course stresses the fact that the simulation of models that appear to be simple often requires significant effort. A well-known example is the Coulomb friction model, which cannot be used for simulation, but must be reformulated with an extra state as in the Dahl or LuGre models, or with a dead-zone as in the Karnopp model. In hydraulics, the square-root characteristic of turbulent orifice flow is problematic in combination with automatic step-length control, and should be regularized with a linear laminar characteristic close to zero flow. The students are given computer assignments in which they develop simulators for a motor and load with elastic transmission and friction, for a fluid transmission line, and for the landing of a helicopter on a ship. Numerical integration methods for use in simulation are presented and analyzed, including explicit and implicit Runge-Kutta methods, backwards differentiation formula methods, and characteristic properties of various numerical schemes.

The main idea of the course is to provide students with sufficient modeling background to design controllers and simulators for a wide range of processes. An interesting observation is the current trend that new products 
and systems are often developed by a team of experts with different backgrounds. To prepare students for this situation, the course employs standard terminology and notation from different engineering disciplines. We deliberately break from the tradition in the control literature, where the emphasis is on having a unified formulation specific to automatic control.

The textbook for this course contains more material than is covered in the lectures, so that students can return to the book at a later stage and find additional information about particular subjects. In the student project work and in the Master's thesis work in the fifth year, students have used this textbook to develop advanced mathematical models and simulators for a wide range of processes. The continued use of the textbook is an indication that the new course on modeling and simulation has been successful. More information on the course is found in [6], and a review of [2] can be found in $[7]$.

\section{References}

[1] http://www.itk.ntnu.no/ansatte/Gravdahl_Jan.Tommy/ July, 2004.

[2] O. Egeland and J.T. Gravdahl, Modeling and Simulation for Automatic Control. Trondheim: Marine Cybernetics, 2002. 
[3] J. Craig, Introduction to Robotics: Mechanics \& Control. AddisonWesley, 1986.

[4] M. Spong and M. Vidyasagar, Robot Dynamics and Control. John Wiley \& Sons, 1989.

[5] L. Sciavicco and B. Siciliano, Modelling and Control of Robot Manipulators. London: Springer, 2000.

[6] http://www.itk.ntnu.no/ansatte/Egeland_Olav/July, 2004.

[7] D.S. Bernstein, "Review of 'Modeling and Simulation for Automatic Control'," Control Systems Magazine, vol. 23, pp. 87-88, Oct. 2003. 


\section{Author biographies}

Jan Tommy Gravdahl graduated as siv.ing. in 1994 and dr.ing. in 1998 in electrical engineering at the Norwegian University of Science and Technology, Trondheim, Norway, where he became associate professor in 2001. He is the author of the monograph Compressor surge and rotating stall: modeling and control (Springer-Verlag), and he received the IEEE Transactions on Control Systems Technology Outstanding Paper Award in 2000. His research interests include modeling and nonlinear control of mechanical systems with applications to compressors and small satellites.

Olav Egeland graduated as siv.ing. in 1984 and dr.ing. in 1987 in electrical engineering at the Norwegian University of Science and Technology, Trondheim, Norway, where he became assistant professor in 1987 and professor of Engineering Cybernetics in 1989. In the academic year 1988-89 he was a visiting scientist at the German Aerospace Research Establishment (DLR) in Oberpfaffenhofen, Germany. He has been associate editor of the IEEE Transactions on Automatic Control and the European Journal of Control. He received the Automatica Prize Paper Award in 1996 and the IEEE Transactions on Control Systems Technology Outstanding Paper Award in 2000. His research interests include modeling and nonlinear control of mechanical systems with applications to robotics and marine systems. 